\title{
Characterization of naturally occurring pentacyclic triterpenes as novel inhibitors of deubiquitinating protease USP7 with anticancer activity in vitro
}

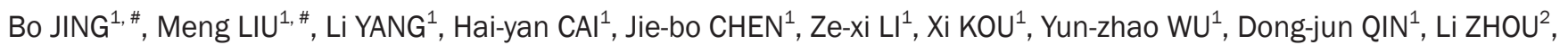 \\ Jin $\mathrm{JIN}^{1}$, Hu LEI ${ }^{1}$, Han-zhang $\mathrm{XU}^{1}$, Wei-wei WANG ${ }^{1, *}$, Ying-li WU ${ }^{1, *}$ \\ ${ }^{1}$ Hongqiao International Institute of Medicine, Shanghai Tongren Hospital/Faculty of Basic Medicine, Chemical Biology Division of \\ Shanghai Universities E-Institutes, Key Laboratory of Cell Differentiation and Apoptosis of the Chinese Ministry of Education, Shanghai \\ Jiao Tong University School of Medicine, Shanghai 200025, China; ${ }^{2}$ Department of Hematology, Rui-Jin Hospital, Shanghai Jiao-Tong \\ University School of Medicine, Shanghai 200025, China
}

\begin{abstract}
Deubiquitinating protease USP7 is a promising therapeutic target for cancer treatment, and interest in developing USP7 inhibitors has greatly increased. In the present study, we reported a series of natural pentacyclic triterpenes with USP7 inhibitory activity in vitro. Among them, both the ursane triterpenes and oleanane triterpenes were more active than the lupine triterpenes, whereas ursolic acid was the most potent with $\mathrm{IC}_{50}$ of $7.0 \pm 1.5 \mu \mathrm{mol} / \mathrm{L}$. Molecular docking studies showed that ursolic acid might occupy the ubiquitin binding pocket of USP7, with the 17-carboxyl group and 3-hydroxyl group playing a vital role in the USP7-ursolic acid interaction. Using the cellular thermal shift assay, we demonstrated that ursolic acid interacted with USP7 in RPMI8226 human myeloma cells. Ursolic acid dose-dependently inhibited the proliferation of the myeloma cells with $\mathrm{IC}_{50}$ of $6.56 \mu \mathrm{mol} / \mathrm{L}$, accompanied by reductions in USP7 substrates such as MDM2, UHRF1 and DNMT1. Overexpression of USP7 partially, but significantly attenuated ursolic acid-induced cell death as well as downregulation of MDM2, UHRF1 and DNMT1. In conclusion, we demonstrate for the first time that pentacyclic triterpenes represent a novel scaffold for developing USP7 inhibitors and that USP7 inhibition contributes to the anti-cancer effect of ursolic acid.
\end{abstract}

Keywords: USP7; pentacyclic triterpenes; ursolic acid; anticancer agent; RPMI8226 human myeloma cells; UHRF1

Acta Pharmacologica Sinica (2018) 39: 492-498; doi: 10.1038/aps.2017.119; published online 23 Nov 2017

\section{Introduction}

Ubiquitination is a highly dynamic process orchestrated by a series of conjugating enzymes ${ }^{[1]}$. Deubiquitylating enzymes (DUBs), which include 5 structurally different DUB enzyme families, can deconjugate ubiquitin from polyubiquitylated proteins $^{[2]}$. Ubiquitin-specific enzymes (USPs) are the largest subfamily of DUBs, with more than 60 members in humans ${ }^{[3,4]}$. USPs such as USP1, USP2, USP7, USP9x and USP14 have been found to be closely correlated with the development of cancer ${ }^{[3-7]}$. Among them, USP7 has received special attention. The inhibition of USP7 is predicted to destabilize MDM2 and stabilize p53, which promotes the cell cycle arrest and

\footnotetext{
\# These authors contributed equally to this work.

* To whom correspondence should be addressed.

E-mail wuyingli@shsmu.edu.cn (Ying-li WU); weiweiwang@shsmu.edu.cn (Wei-wei WANG)

Received 2017-04-05 Accepted 2017-07-21
}

apoptosis of cancer cells ${ }^{[8]}$. Moreover, many cancer-related proteases have been reported as additional substrates of USP7, such as UHRF1, DNMT1, claspin, FOXO4 and PTEN ${ }^{[9-13]}$. The overexpression of USP7 has been observed in prostate cancer, myeloma cells, neuroblastoma, breast cancer, and ovarian cancer $^{[7,14-17]}$, while inhibiting USP7 can inhibit proliferation and induce cell death in cancer cells. Therefore, the development of USP7 inhibitors has attracted great attention for the treatment of cancer. Although many small-molecule USP7 inhibitors have been reported, no natural product has been reported as an inhibitor of USP7 to date ${ }^{[18]}$.

Pentacyclic triterpenes and their derivatives occur widely in the plant kingdom and have been reported to have a variety of biological activities, such as antitumor, anti-inflammatory, antiviral and antioxidant activities ${ }^{[19,20]}$. For example, ursolic acid has been shown to inhibit the proliferation of a variety of cancers, such as breast, prostate, and liver cancer and leuke- 
mia. In addition, multiple signaling pathways including the STAT3, NF-kB, PTEN, and TRAIL pathways have been shown to be involved in the anticancer effect of ursolic acid ${ }^{[21,22]}$. We previously reported that CDDO-Me had USP7 inhibitory activity. This result prompted us to investigate whether deubiquitylating enzymes play a role in the action of pentacyclic triterpenes such as ursolic acid.

In the present study, we demonstrated for the first time that a series of naturally occurring pentacyclic triterpenes inhibit USP7 activity by directly interacting with USP7. Among them, ursolic acid (1) shows inhibitory activity against USP7 in vitro and in cells. In addition, the inhibition of USP7 contributes to the apoptosis-inducing effect of ursolic acid in myeloma cells. Our results suggest that natural pentacyclic triterpenes represent a novel scaffold for developing USP7 inhibitors and that USP7 is a novel target involved in the anticancer effect of ursolic acid.

\section{Materials and methods Chemical reagents}

Natural pentacyclic triterpenes (compounds 1-13, purity>98\%) were purchased from Herbpurify (Chengdu, China). All solvents were purified and dried by standard procedures and stored over a suitable drying agent prior to use. Unless otherwise noted, all reagents were purchased from commercial sources and used without further purification.

For chemical synthesis, the reactions were monitored by thin layer chromatography on plates coated with silica gel. ${ }^{1} \mathrm{H}$ NMR data were acquired with $\mathrm{CDCl}_{3}$ as the solvent on a Bruker DMX 500 or a JEOL ECA-400 spectrometer. The chemical shifts $(\delta)$ were expressed in parts per million (ppm) relative to TMS (tetramethylsilane). Spin-spin coupling constants $(J)$ were measured directly from the spectra and given in Hz. Exact mass (HR-MS) spectra were recorded on a Thermo Fisher Scientific LTQ FT Ultra (USA). Purity was based on the integrated UV chromatogram (254 nm), and compounds 14-15 had purity $\geq 95 \%$ as verified by HPLC.

\section{Cell culture}

Human myeloma cells (RPMI8226) were purchased from the American Type Culture Collection (ATCC, Manassas, VA, USA) and cultured in RPMI-1640 (Gibco, Foster City, CA, USA) supplemented with $10 \%(w / v)$ fetal bovine serum (FBS; Gibco) and $1 \%$ penicillin-streptomycin (Gibco). The cell lines were maintained at $37^{\circ} \mathrm{C}$ in a humidified atmosphere with $5 \% \mathrm{CO}_{2}$.

\section{In vitro gel-based USP7 activity assay}

USP7 ( $5 \mathrm{nmol} / \mathrm{L}$ ) was incubated with compounds for $10 \mathrm{~min}$ at $37{ }^{\circ} \mathrm{C}$ in reaction buffer $(50 \mathrm{mmol} / \mathrm{L}$ Tris- $\mathrm{HCl} \mathrm{pH} 8.0,20$ $\mathrm{mmol} / \mathrm{L} \mathrm{NaCl}, 2 \mathrm{mmol} / \mathrm{L}$ DTT). Then, GST-UBA52 (3.92 $\mu \mathrm{mol} / \mathrm{L}$, final concentration) was added and incubated for another $45 \mathrm{~min}$ at $37^{\circ} \mathrm{C}$. The reaction was terminated by adding loading buffer and boiling on a heat block. The proteins were separated by $12 \%$ SDS-PAGE and visualized with Coomassie brilliant blue (G250). The density of the bands was measured with Quantity One software (version 4.69, Bio-Rad,
CA, USA), and $\mathrm{IC}_{50}$ was determined using GraphPad software (version Prism 5 Demo, GraphPad Software, Inc, CA, USA). All experiments were repeated three times.

\section{Purification of GST-UBA52}

The pET28a(+)-UBA52 plasmid was transfected into $E$ coli BL21, and the expression of UBA52 was induced with 0.5 $\mathrm{mmol} / \mathrm{L}$ isopropyl- $D$-thiogalactoside (IPTG) at $16^{\circ} \mathrm{C}$ for $12 \mathrm{~h}$. Cell pellets were resuspended in lysis buffer (300 mmol/L NaCl, $50 \mathrm{mmol} / \mathrm{L} \mathrm{PBS} \mathrm{pH} \mathrm{8.0,} 10 \mathrm{mmol} / \mathrm{L}$ imidazole, $10 \mathrm{mmol} / \mathrm{L} \beta$-mercaptoacetic ethanol and $10 \mathrm{mmol} / \mathrm{L}$ glycerol) and sonicated. His-tagged proteins were purified on Ni-NTA-agarose and eluted with $250 \mathrm{mmol} / \mathrm{L}$ imidazole in $300 \mathrm{mmol} / \mathrm{L} \mathrm{NaCl}, 50 \mathrm{mmol} / \mathrm{L}$ PBS pH 8.0, $10 \mathrm{mmol} / \mathrm{L}$ $\beta$-mercaptoacetic ethanol and 10\% glycerol.

\section{Cellular thermal shift assay (CETSA)}

RPMI8226 cells were harvested and washed with PBS. The cells were diluted in PBS supplemented with a complete protease inhibitor cocktail. The cell suspensions were frozen and thawed three times using liquid nitrogen. The soluble fraction (lysate) was separated from the cell debris by centrifugation at $20000 \times g$ for $20 \mathrm{~min}$ at $4^{\circ} \mathrm{C}$. The cell lysates were diluted with PBS and divided into two aliquots, one treated with DMSO and the other with diluted ursolic acid. After $30 \mathrm{~min}$ of incubation at room temperature, the respective lysates were divided into smaller $(50 \mu \mathrm{L})$ aliquots and heated individually at different temperatures for $3 \mathrm{~min}$ (Veriti Thermal Cycler, Applied Biosystems/Life Technologies) followed by cooling for $3 \mathrm{~min}$ at room temperature. The appropriate temperatures were determined in preliminary CETSA experiments (data not shown). The heated lysates were centrifuged at $20000 \times g$ for $20 \mathrm{~min}$ at $4{ }^{\circ} \mathrm{C}$ to separate the soluble fractions from the precipitates. The supernatants were transferred to new microtubes and analyzed by sodium dodecyl sulfate polyacrylamide gel electrophoresis (SDS-PAGE) followed by Western blotting analysis.

\section{Cell apoptosis assays}

Cell apoptosis was measured with the Annexin V Apoptosis Detection Kit (BD Pharmingen, USA) following the manufacturer's instructions. Annexin V-positive and propidium iodide-negative cells were considered to be in the early apoptotic phase, and cells with positive staining for both Annexin $\mathrm{V}$ and propidium iodide were considered to be in the stages of late apoptosis or necrosis. All data were collected, stored, and analyzed with the LYSIS II software (BD Biosciences). All experiments were repeated three times.

\section{Western blotting}

Cells were washed with PBS and lysed with lysis buffer (50 $\mathrm{mmol} / \mathrm{L}$ Tris- $\mathrm{HCl}, \mathrm{pH} 6.8,100 \mathrm{mmol} / \mathrm{L}$ DTT, 2\% SDS, 10\% glycerol). Cell lysates were centrifuged at $20000 \times g$ for $10 \mathrm{~min}$, and the proteins in the supernatants were quantified. Equal amounts of protein extracts were loaded onto an $8 \%$ to $12 \%$ SDS-polyacrylamide gel, electrophoresed, and transferred 
to a nitrocellulose membrane (Bio-Rad). The blots were stained with $0.2 \%$ Ponceau $S$ red to ensure equal protein loading. After blocking with 5\% nonfat milk in PBS, the membranes were probed with antibodies against USP7, MDM2, caspase-3, PARP1, UHRF1, and DNMT1. The signals were detected with a chemiluminescence PhototopeHRP Western Blot Detection Kit (Cell Signaling) according to the manufacturer's instructions. All experiments were repeated three times with similar results.

\section{Molecular docking}

Molecular docking was carried out using the software AutoDock4.2. The X-ray structure of the USP7 catalytic domain (PDB ID: 4M5W) was retrieved from the Protein Data Bank (http://www.rcsb.org/pdb) for the docking calculations. Water and bromide ions were all removed. To prepare for both the protein and the small molecule, first, all hydrogens were added; then, Gasteiger charges were computed, and non-polar hydrogens were merged. The active site was defined by a grid box of $70 \times 70 \times 70$ points with a grid spacing of $0.375 \AA$ using AutoGrid4. The box was centered on the residue Tyr514 in the crystal structure of USP7. The protein was considered rigid for the docking study. The docking parameters were set as follows: ga_pop_size $=150$ (number of individuals in population) and ga_ run=100 (the number of dockings that were performed). The default values in the software were used for other parameters. Protein-ligand interactions were handled using the Lamarckian genetic algorithm. Finally, the conformation was selected according to the predicted binding free energy.

\section{Statistical analysis}

The data are presented as the mean $\pm S D$ of at least three independent experiments. Student's $t$-tests were performed using the GraphPad Prism 5.0 software (GraphPad Software); $P<0.05$ was considered statistically significant.

\section{Results}

\section{Pentacyclic triterpenes have USP7 inhibitory activity}

Thirteen natural pentacyclic triterpenes (Figure 1A) were biologically evaluated for inhibitory activity against USP7 in vitro. Based on their structural skeleton, pentacyclic triterpenes can be classified into three major types: (a) the ursane type of triterpenes (compounds 1-4); (b) the oleanane type of triterpenes (compounds 5-11); and (c) the lupane type of triterpenes (compounds 12, 13). As shown in table 1, ursolic acid (1) was shown to be the most potent of these analogues, with an $\mathrm{IC}_{50}$ value as low as $7.0 \mu \mathrm{mol} / \mathrm{L}$ for USP7; oleanolic acid (5) showed similar activity to that of ursolic acid (1); corosolic

A
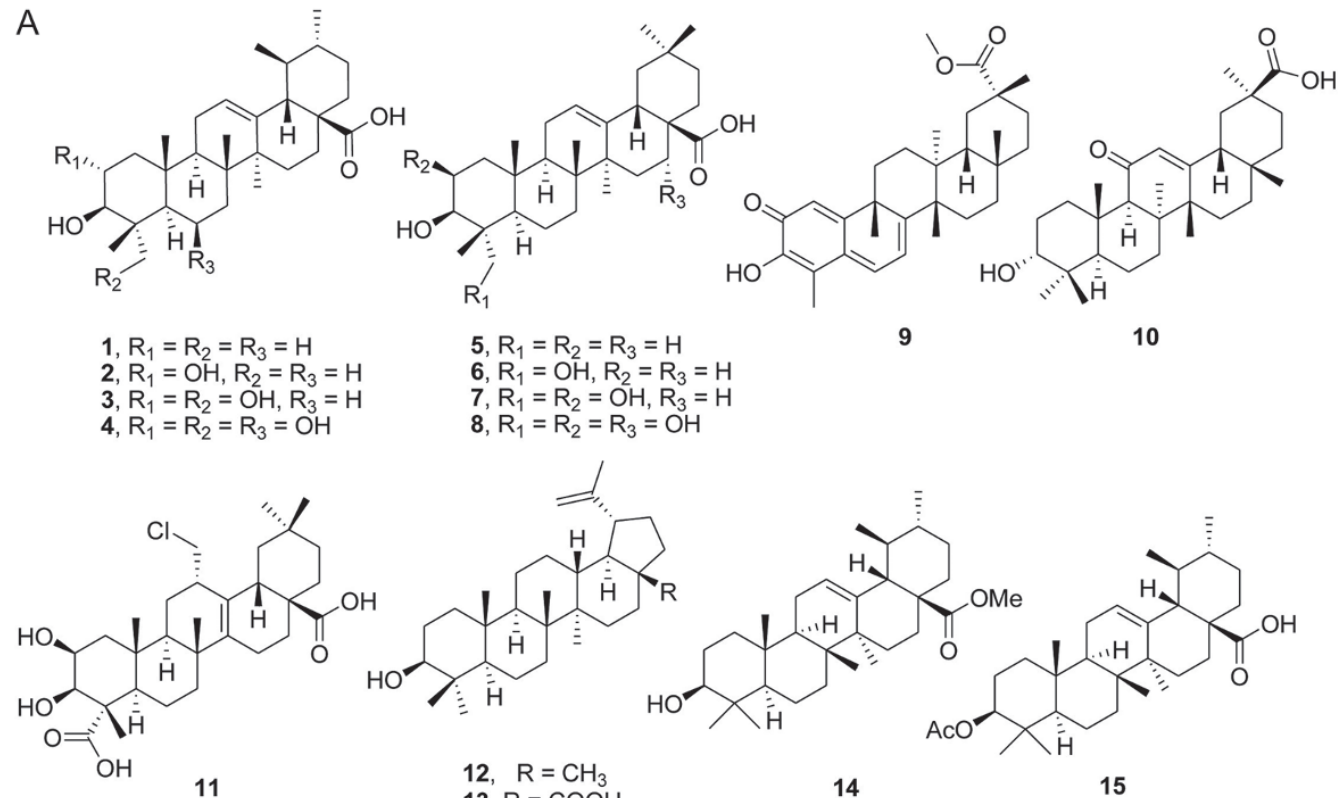

12, $\mathrm{R}=\mathrm{CH}_{3}$
13, $\mathrm{R}=\mathrm{COOH}$

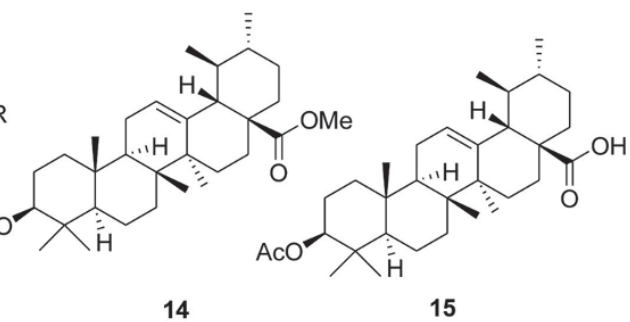

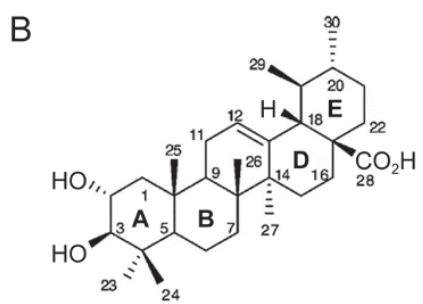

Figure 1. Chemical structure of compounds. (A) Structure of naturally occurring pentacyclic triterpenes (compounds 1-13) and ursolic acid derivatives (compounds 14-15). (B) The numbering system of the chemical structure of compound 1 (ursolic acid). 
Table 1. $I C_{50}$ values ( $\left.\mu \mathrm{mol} / \mathrm{L}\right)$ of naturally occurring pentacyclic triterpenes and ursolic acid derivatives for the inhibition of USP7 in vitro.

\begin{tabular}{lll}
\hline Compd & \multicolumn{1}{c}{ Name } & $\mathrm{IC}_{50}(\mu \mathrm{mol} / \mathrm{L})$ \\
\hline 1 & Ursolic acid & $7.0 \pm 1.5$ \\
2 & Corosolic acid & $22.7 \pm 2.1$ \\
3 & Asiatic acid & $\mathrm{NA}^{\mathrm{a}}$ \\
4 & Madecassic acid & $\mathrm{NA}^{\mathrm{a}}$ \\
5 & Oleanolic acid & $12.5 \pm 1.0$ \\
6 & Hederagenin & $33.5 \pm 1.0$ \\
7 & Bayogenin & $\mathrm{NA}^{\mathrm{a}}$ \\
8 & Polygalacic acid & $\mathrm{NA}$ \\
9 & Pristimerin & $21.1 \pm 3.2$ \\
10 & Glycyrrhetic acid & $\mathrm{NA}$ \\
11 & Senegenin & $\mathrm{NA}^{\mathrm{a}}$ \\
12 & Lupeol & $\mathrm{NA}^{\mathrm{a}}$ \\
13 & Betulinic acid & $33.3 \pm 2.6$ \\
14 & - & $\mathrm{NA}^{\mathrm{a}}$ \\
15 & - & $\mathrm{NA}^{\mathrm{a}}$ \\
\hline
\end{tabular}

${ }^{a} \mathrm{NA}=$ no activity $\left(\mathrm{IC}_{50}>50 \mu \mathrm{mol} / \mathrm{L}\right)$.

acid (2), hederagenin (6), pristimerin (9) and betulinic acid (13) showed mild inhibition of USP7; and the other compounds showed no inhibition. These results revealed that, among the different structural skeletons, both the ursane triterpenes and the oleanane triterpenes were more active than the lupine triterpenes (compounds 1 and 5 vs 13). The number and position of the hydroxyl groups of triterpenes on ring A (for the numbering system of the chemical structures, see Figure 1B) seemed to have an important influence on their potency. Introducing a 2-hydroxyl group and 23-hydroxyl group led to an obvious decrease in the inhibition of USP7 (compound $\mathbf{1}$ vs 2 and 3). The same tendency was also observed in oleanane triterpenes (compound 5 vs $\mathbf{6}$ and 7).

\section{Molecular docking of USP7-ursolic acid}

To elucidate the structural basis of USP7 inhibition by pen- tacyclic triterpenes, the USP7-ursolic acid complex was modeled based on the crystal structure of the USP7 catalytic domain (PDB ID: 4M5W). Our results showed that ursolic acid occupied the ubiquitin binding pocket of USP7 (Figure 2). The skeleton of ursolic acid hydrophobically interacts with residues Met328, Tyr367, Ala369 and Val393. Two hydrogen bonds in the complex structure are the most notable: the 17-carboxyl group of ursolic acid could form hydrogen bonds with Glu371, and the 3-hydroxyl group could form a hydrogen bond with Gln351. This computational result gave a reasonable explanation for the lack of activity of compounds $\mathbf{1 4}$ and 15. Both the theoretical analyses and experimental examination revealed that the 17-carboxyl group and 3-hydroxyl group played vital roles in the USP7-ursolic acid interaction.

\section{Ursolic acid interacts with USP7 in myeloma cells}

USP7 is expressed at high levels in multiple myeloma cells and the inhibition of USP7 inhibits cell proliferation and induces cell death in myeloma cells ${ }^{[16]}$. To determine whether the USP7-inhibitory effect of pentacyclic triterpenes observed in vitro existed in cells, we selected ursolic acid (1) for further investigation using RPMI8226 myeloma cells as a cell model. First, we used the cellular thermal shift assay (CETSA) to evaluate drug binding to target proteins in cells ${ }^{[17,18]}$. Aliquots of RPMI8226 cell lysate incubated with DMSO or ursolic acid were heated to different temperatures. After cooling, the samples were centrifuged to separate the soluble fractions from precipitated proteins. We then quantified the presence of USP7 in the soluble fraction by Western blotting. Compared with the DMSO-treated cell lysate, ursolic acid treatment led to an obvious increase in the USP7 protein level in the supernatant (Figure 3A-B). Consistent with these results, ursolic acid could increase the thermal stability of USP7 in a dosedependent manner (Figure 3C-D). To investigate the selectivity of ursolic acid, we examined its effect on the thermal stability of several other cysteine proteases, such as USP8, USP18, USP44, USP47, caspase- 3 and caspase-8. The results showed that ursolic acid did not affect the thermal stability of these

A
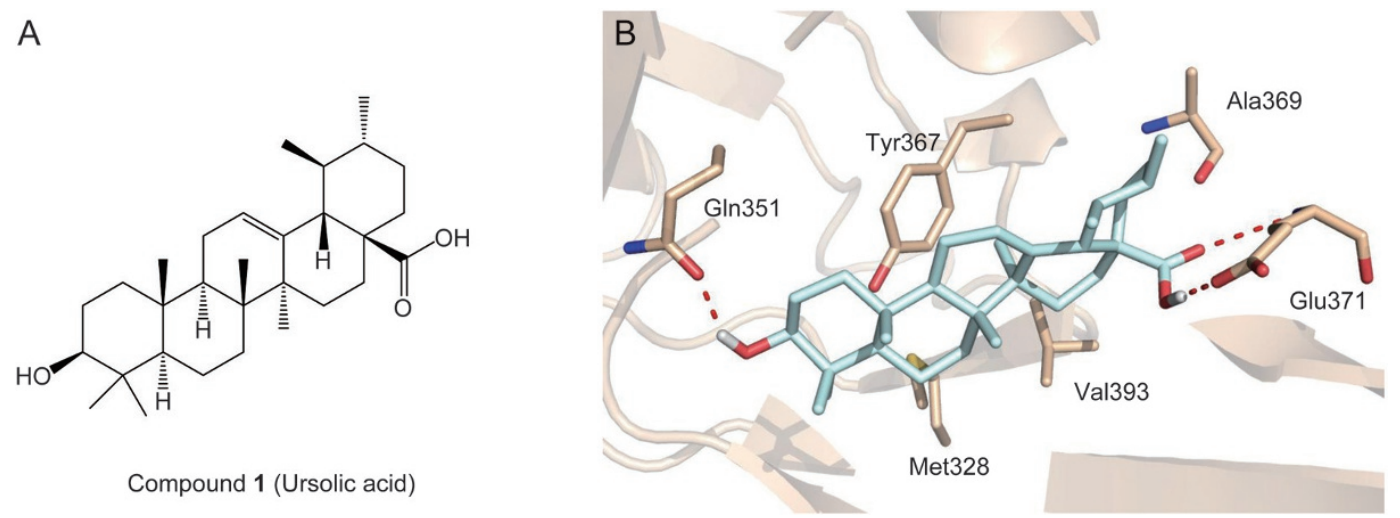

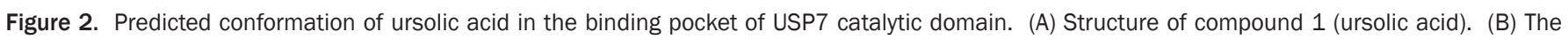

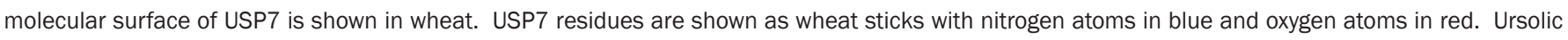
acid is shown in pale cyan sticks. 

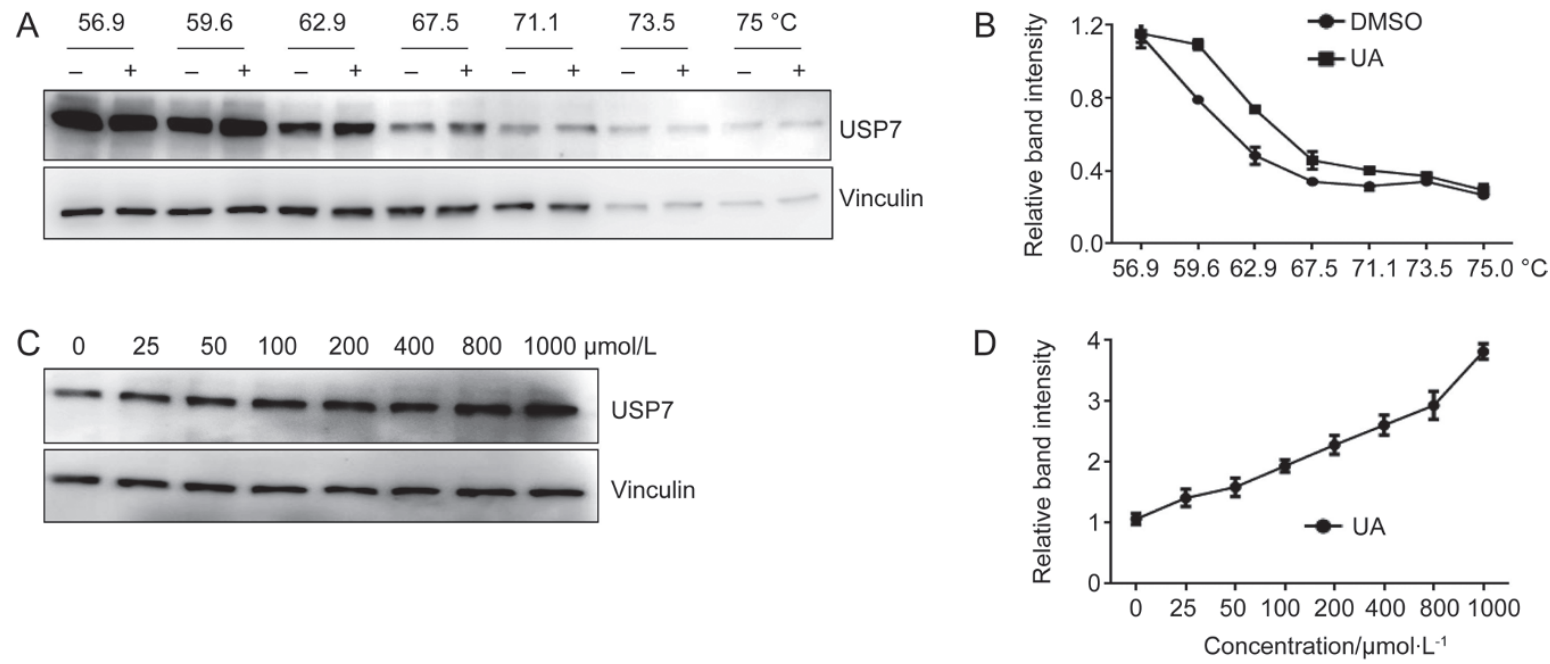

Figure 3. Ursolic acid interacts with USP7 in RPMI8226 myeloma cells. (A-B) CETSA was performed on RPMI8226 cells as described in the Materials and Methods. The stabilizing effects of ursolic acid on USP7 and vinculin at different temperatures (A) and different doses (C) were evaluated by Western blotting analysis. The intensity of the USP7 bands was quantified using the Quantity One software (B, D).

cysteine proteases (Supplementary Figure S1).

\section{Ursolic acid inhibits USP7 activity in myeloma cells}

The inhibition of USP7 can inhibit proliferation and induce apoptosis in myeloma cells. As expected, ursolic acid treatment for $24 \mathrm{~h}$ inhibited the proliferation of RPMI8226 cells, with an $\mathrm{IC}_{50}$ of $26.56 \mu \mathrm{mol} / \mathrm{L}$ (Figure $4 \mathrm{~A}$ ). The percentage of apoptotic cells was evaluated by Annexin V/PI double staining. As shown in Figure 4B, ursolic acid induced apoptosis in RPMI8226 cells in a dose- and time-dependent manner. Consistent with these findings, ursolic acid treatment led to the activation of caspase- 3 and cleavage of PARP1, indicating the activation of apoptosis (Figure 4B). Moreover, in parallel with the inhibition of USP7, ursolic acid treatment at $20 \mu \mathrm{mol} / \mathrm{L}$ resulted in a decrease in UHRF1 and DNMT1, two substrates of USP7 (Figure 4B). These results indicate that ursolic acid inhibits USP7 activity and induces apoptosis in myeloma cells.

\section{Overexpression of USP7 partially inhibits the anticancer effect of ursolic acid}

To investigate the role of USP7 in ursolic acid-induced cell death, a USP7 plasmid or control vector was stably transfected into RPMI8226 cells (Figure 4C), and the cells were treated with ursolic acid. As shown in Figure 4D, compared with the control cells, the overexpression of USP7 partially but significantly inhibited ursolic acid-induced apoptosis $(P<0.05)$. Consistent with this result, the cleavage of caspase- 3 and PARP1 was also inhibited (Figure 4E). Interestingly, the ursolic acidinduced decreases in MDM2, UHRF1 and DNMT1 were also partially blocked by the overexpression of USP7 (Figure 4E). These data indicate that the inhibition of USP7 contributes to ursolic acid-induced apoptosis.

\section{Discussion}

Pentacyclic triterpenes are a broad family of compounds with a variety of activities. However, whether this kind of compound exhibits activity against deubiquitinating enzymes was not known. In the present study, we demonstrated for the first time that natural pentacyclic triterpenes are a novel type of scaffold for the development of USP7 inhibitors. Using ursolic acid as a model compound, we demonstrated that targeting USP7 contributes to the anti-cancer effect of ursolic acid.

Ursolic acid exhibits wide-ranging anti-inflammatory and anticancer activities. In addition, many signaling pathways such as the Nrf2, NF-KB, STAT3, and AKT pathways can be modulated by ursolic acid ${ }^{[21]}$. However, the direct target of ursolic acid is not clear. The identification of USP7 as a novel target of ursolic acid may explain some of the activities of ursolic acid. For instance, ursolic acid has been shown to upregulate the expression of $\mathrm{p} 53^{[22]}$. This upregulation may result from the USP7 inhibition-induced degradation of MDM2; ursolic acid could reduce the DNA methyltransferase DNMT1, which might be related to the inhibition of USP7 $7^{[23]}$. Moreover, as USP7 has other substrates such as PTEN, FOXO4 and UHRF1, it is possible that ursolic acid could regulate these protein-related signaling pathways and show broad activities against cancers with dysregulation of these pathways.

Pentacyclic triterpenoids have highly diverse structures ${ }^{[24]}$. Our data showed that some of the tested pentacyclic triterpenoids had USP7 inhibitory activity. The SAR analysis suggested that the number and position of hydroxyl groups of triterpenes in ring A seemed to have an important influence on potency. Consistent with this hypothesis, the conversion of the 17-carboxyl group and 3-hydroxyl group of ursolic acid to ester groups (compounds $\mathbf{1 4}$ and 15) resulted in a significant loss of potency (compound 1 vs $\mathbf{1 4}$ and 15). These data suggest that the interaction between pentacyclic triterpenoids and USP7 has unique stereospecificity. In addition, this characteristic may allow the development of novel derivatives that are more selective against USP7. 
A

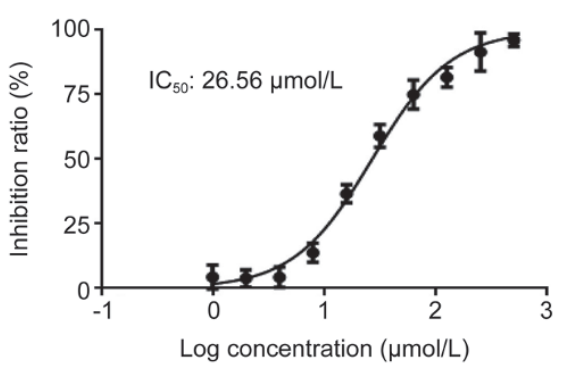

C
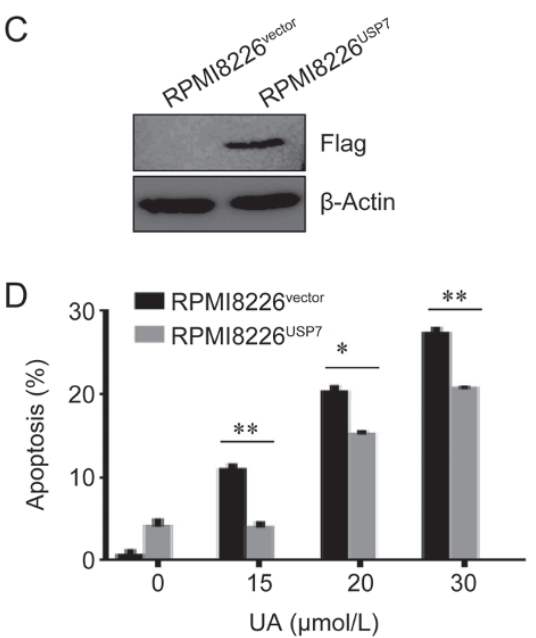

B

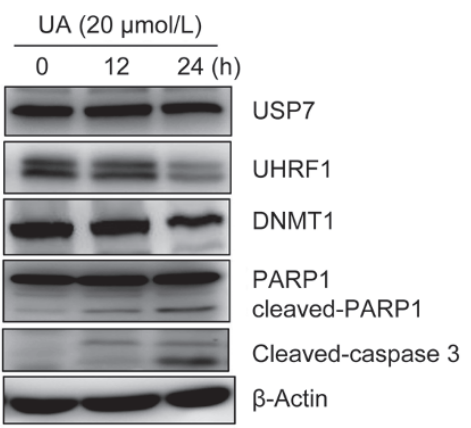

E

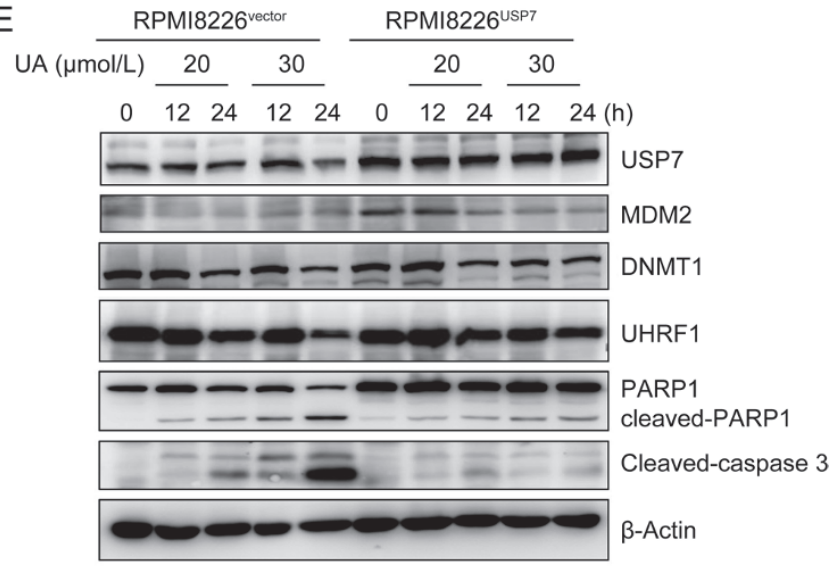

Figure 4. Overexpression of USP7 partially inhibits ursolic acid-induced apoptosis in RPMI8226 cells. (A-B) RPMI8226 cells were treated with ursolic acid at different doses for $24 \mathrm{~h}$. Cell proliferation was evaluated by the CCK8 assay (A), and the indicated proteins were examined by Western blotting (B). (C) USP7 plasmid or the control vector was stably transfected into RPMI8226 cells, and the indicated proteins were detected by Western blotting. (DE) The transfected cells were treated with ursolic acid for different times. Annexin V-positive cells were evaluated by flow cytometry analysis (D), and the indicated proteins were examined by Western blotting $(E)$. ${ }^{*} P<0.05,{ }^{* *} P<0.01$.

Currently, the USP7 inhibitory effect of the tested pentacyclic triterpenoids is not very potent. Thus, using synthetic pentacyclic triterpenoids to develop novel potent USP7 inhibitors is a promising strategy. Crystalline ursolic acid of high purity is easily obtained, and many ursolic acid derivatives have been synthesized ${ }^{[25,26]}$. However, most of these synthesized compounds are not directed to a clear target. Therefore, whether these ursolic acid derivatives have USP7 inhibitory activities warrants further investigation.

In conclusion, to our knowledge, this report is the first to show that natural pentacyclic triterpenes have USP7 inhibitory activity and that pentacyclic triterpenes represent a novel scaffold for designing novel USP7 inhibitors. Using ursolic acid as a model compound, we demonstrate that USP7 is a novel target of ursolic acid, which may broaden the use of ursolic acid in USP7-related cancers.

\section{Acknowledgments}

This work was supported in part by grants from the National Key Research and Development Program of China (№ 2017YFA0505200), the National Basic Research Program of China (973 Program) (№ 2015CB910403), the National Natu- ral Science Foundation of China (№ 21602133, 81570118, 81570112), and the Science and Technology Committee of Shanghai (№ 15401901800 and 13ZR1456900).

\section{Author contribution}

Ying-li WU and Wei-wei WANG designed the research; Bo JING, Meng LIU, Jie-bo CHEN, Ze-xi LI, Xi KOU, Yun-zhao WU, Hai-yan CAI, Dong-jun QIN, and Jin JIN performed the research; Li YANG, Li ZHOU, and Hu LEI contributed new reagents or analytic tools; Han-zhang XU, Wei-wei WANG and Ying-li WU analyzed the data; and Ying-li WU and Weiwei WANG wrote the paper.

\section{References}

1 Gallo LH, Ko J, Donoghue DJ. The importance of regulatory ubiquitination in cancer and metastasis. Cell Cycle 2017; 16: 634-48.

2 Eletr ZM, Wilkinson KD. Regulation of proteolysis by human deubiquitinating enzymes. Biochim Biophys Acta 2014; 1843: 11428.

3 Garcia-Santisteban I, Peters GJ, Giovannetti E, Rodriguez JA. USP1 deubiquitinase: cellular functions, regulatory mechanisms and emerging potential as target in cancer therapy. Mol Cancer 2013; 12: 91. 
4 Wang CL, Wang JY, Liu ZY, Ma XM, Wang XW, Jin H, et al. Ubiquitinspecific protease $2 a$ stabilizes MDM4 and facilitates the p53mediated intrinsic apoptotic pathway in glioblastoma. Carcinogenesis 2014; 35: 1500-9.

5 Potu H, Peterson LF, Kandarpa M, Pal A, Sun H, Durham A, et al. Usp9x regulates Ets-1 ubiquitination and stability to control NRAS expression and tumorigenicity in melanoma. Nat Commun 2017; 8: 14449.

6 Tian Z, D'Arcy P, Wang X, Ray A, Tai YT, Hu Y, et al. A novel small molecule inhibitor of deubiquitylating enzyme USP14 and UCHL5 induces apoptosis in multiple myeloma and overcomes bortezomib resistance. Blood 2014; 123: 706-16.

7 Qin D, Wang W, Lei H, Luo H, Cai H, Tang C, et al. CDDO-Me reveals USP7 as a novel target in ovarian cancer cells. Oncotarget 2016; 7: 77096-109.

8 Colland F, Formstecher E, Jacq X, Reverdy C, Planquette C, Conrath $\mathrm{S}$, et al. Small-molecule inhibitor of USP7/HAUSP ubiquitin protease stabilizes and activates $p 53$ in cells. Mol Cancer Ther 2009; 8: 2286-95.

9 Morotti A, Panuzzo C, Crivellaro S, Pergolizzi B, Familiari U, Berger AH, et al. BCR-ABL disrupts PTEN nuclear-cytoplasmic shuttling through phosphorylation-dependent activation of HAUSP. Leukemia 2014; 28 : 1326-33.

10 Qin W, Leonhardt H, Spada F. Usp7 and Uhrf1 control ubiquitination and stability of the maintenance DNA methyltransferase Dnmt1. J Cell Biochem 2011; 112: 439-44.

11 Felle M, Joppien S, Nemeth A, Diermeier S, Thalhammer V, Dobner $\mathrm{T}$, et al. The USP7/Dnmt1 complex stimulates the DNA methylation activity of Dnmt1 and regulates the stability of UHRF1. Nucleic Acids Res 2011; 39: 8355-65.

12 van der Horst A, de Vries-Smits AM, Brenkman AB, van Triest MH, van den Broek N, Colland $\mathrm{F}$, et al. FOXO4 transcriptional activity is regulated by monoubiquitination and USP7/HAUSP. Nat Cell Biol 2006; 8: 1064-73.

13 Faustrup H, Bekker-Jensen S, Bartek J, Lukas J, Mailand N. USP7 counteracts SCFbetaTrCP- but not APCCdh1-mediated proteolysis of Claspin. J Cell Biol 2009; 184: 13-9.

14 Wang Q, Ma S, Song N, Li X, Liu L, Yang S, et al. Stabilization of histone demethylase PHF8 by USP7 promotes breast carcinogenesis. J Clin Invest 2016; 126: 2205-20.

15 Tavana O, Li D, Dai C, Lopez G, Banerjee D, Kon N, et al. HAUSP deubiquitinates and stabilizes N-Myc in neuroblastoma. Nat Med 2016; 22: 1180-6.

16 Chauhan D, Tian Z, Nicholson B, Kumar KG, Zhou B, Carrasco R, et al. A small molecule inhibitor of ubiquitin-specific protease-7 induces apoptosis in multiple myeloma cells and overcomes bortezomib resistance. Cancer Cell 2012; 22: 345-58.

17 Chen ST, Okada M, Nakato R, Izumi K, Bando M, Shirahige K. The deubiquitinating enzyme USP7 regulates androgen receptor activity by modulating Its binding to chromatin. J Biol Chem 2015; 290: 2171323.

18 Weinstock J, Wu J, Cao P, Kingsbury WD, McDermott JL, Kodrasov MP, et al. Selective dual inhibitors of the cancer-related deubiquitylating proteases USP7 and USP47. ACS Med Chem Lett 2012; 3: 789-92.

19 Salvador JA, Moreira VM, Goncalves BM, Leal AS, Jing Y. Ursane-type pentacyclic triterpenoids as useful platforms to discover anticancer drugs. Nat Prod Rep 2012; 29: 1463-79.

20 Liby KT, Sporn MB. Synthetic oleanane triterpenoids: multifunctional drugs with a broad range of applications for prevention and treatment of chronic disease. Pharmacol Rev 2012; 64: 972-1003.

21 Shanmugam MK, Dai X, Kumar AP, Tan BK, Sethi G, Bishayee A. Ursolic acid in cancer prevention and treatment: molecular targets, pharmacokinetics and clinical studies. Biochem Pharmacol 2013; 85: 1579-87.

22 Prasad S, Yadav VR, Sung B, Reuter S, Kannappan R, Deorukhkar A, et al. Ursolic acid inhibits growth and metastasis of human colorectal cancer in an orthotopic nude mouse model by targeting multiple cell signaling pathways: chemosensitization with capecitabine. Clin Cancer Res 2012; 18: 4942-53.

23 Kim H, Ramirez CN, Su ZY, Kong AN. Epigenetic modifications of triterpenoid ursolic acid in activating Nrf2 and blocking cellular transformation of mouse epidermal cells. J Nutr Biochem 2016; 33 : 54-62.

24 Zhou M, Zhang RH, Wang M, Xu GB, Liao SG. Prodrugs of triterpenoids and their derivatives. Eur J Med Chem 2017; 131: 222-36.

25 Chen H, Gao Y, Wang A, Zhou X, Zheng Y, Zhou J. Evolution in medicinal chemistry of ursolic acid derivatives as anticancer agents. Eur J Med Chem 2015; 92: 648-55.

26 Markov AV, Logashenko EB, Zenkova MA. Modulation of tumourrelated signaling pathways by natural pentacyclic triterpenoids and their semisynthetic derivatives. Curr Med Chem 2017; 24: $1277-$ 320 . 